\title{
ACTION D'UNE ACIDOSE MÉTABOLIQUE SUR LES TRANSFERTS D'IONS EFFECTUÉS DANS L'OVIDUCTE DE LA POUL.E
}

\author{
B. SAUVEUR \\ avec la collaboration technique de J. Rocard \\ Station de Recherches avicoles, \\ Centre de Recherches de Tours, 37-Nouzilly (France) \\ Institut national de la Recherche agronomique
}

\section{IN'TRODUC'TION}

Deux séries de travaux ont montré que la teneur en électrolytes de l'albumen de l'œuf varie avec 1'équilibre acido-basique général de la poule. Ainsi Hunt (I964), après addition au régime de 2 p. roo de chlorure d'ammonium pendant 56 jours, rapporte les modifications de concentration suivantes pour l'albumen : sodium $-8,5$ p. I00, potassium $+7,7$ p. Ioo, chlore +53 p. Ioo et un abaissement du $\mathrm{pH}$ non chiffré. La seconde série expérimentale a été effectuée récemment dans notre laboratoire (SAUVEUR, I970) ; nous avons enregistré la teneur en ions de 1'albumen 2 jours, puis $\mathrm{I} 2$ et 22 jours après adjonction au régime de base de $3 \mathrm{p}$. Ioo de $\mathrm{NH}_{4} \mathrm{Cl}$ ou de quantités équivalentes de $\left(\mathrm{NH}_{4}\right)_{2} \mathrm{SO}_{4}, \mathrm{HCl}$ et $\mathrm{H}_{2} \mathrm{SO}_{4}$. Il apparaît que la composition du blanc dépend très peu de la durée du traitement mais est sujette à de grandes variations suivant la nature des corps ajoutés au régime. Nous avons pu démontrer en particulier que les modifications constatées tant au niveau de l'œuf qu'à celui du sang (SAUVEUR, I969) sont essentiellement liées à 1'anion qui accompagne les protons ajoutés au régime.

A titre d'exemple les variations de concentrations observées dans l'albumen après I2 jours de traitement avec $3 \mathrm{p}$. Ioo de $\mathrm{NH}_{4} \mathrm{Cl}$ sont les suivantes : sodium - 2 I p. IOO, potassium + II p. IOO, chlore +52 p. IOO, calcium +2 IO p. IOO, bicarbonates $-5^{6} \mathrm{p}$. Ioo, tandis que le $\mathrm{pH}$ chute de $\mathrm{I} 8,5 \mathrm{p}$. Ioo (variations toutes significatives, $p<0$,OoI).

Certaines de ces modifications étant considérables, nous avons voulu déterminer quelle(s) partie(s) de l'oviducte en étai(en)t responsable(s). Nous avons pour cela prélevé des œufs en formation à la sortie du magnum, de l'isthme et de l'utérus (œuf pondu) chez des animaux témoins et d'autres en acidose au chlorure d'ammonium. Il apparaît que le dernier site de transfert d'ions (utérus) est, dans la majorité des 
cas, le seul affecté par l'état d'acidose et que l'importance relative des trois segments considérés peut être largement modifiée par ce traitement.

\section{MATÉRIEL ET MÉTHODES}

\section{Provenance et traitement des ceufs}

Cettc étude a été effectuée avec $4^{8}$ poules Loghorn à crête simple (H. N.) ; I 4 d'entre elles ont reçu un régime de base classique supplémenté avec 3 p. Ioo de $\mathrm{NH}_{4} \mathrm{Cl}$, les 34 autres consommant le régime de base seul. Soixante quatre oufs pondus ont été analysés dont 26 provenaient d'animaux en acirlose.

Les fins de passage de l'cuuf dans le magnum et l'isthme ont été fixées respectivement à 3 h i 5 et $4 \mathrm{~h} 30$ après oviposition cle l'cuf précédent; (dans le $2^{\circ}$ cas les membranes coquillières ont toujours été présentes).

Nous avons ainsi abattu 33 poules au stade 3 h I 5 (dont 6 en acidose) et I 5 à + h 30 (dont 8 en acidose) ; l'ouf en formation a alors été retiré de l'oviducte, traité suivant une méthode déjà décrite (S'Auveur, I970) et les teneurs de l'albumen en $\mathrm{Na}, \mathrm{K}, \mathrm{Ca}$ et $\mathrm{Cl}$ ont été comparées à celles enregistrées clans l'ouf pondu la veille.

\section{Calculs effectués et presentation des données}

A la suite des travaux de Scott, Hughes et Warken (I937), nous pouvons admettre que l'apport d'azote à l'albumen est terminé quand l'œuf a quitté le magnum. Sachant de plus que les cendres apportées dans l'isthme et l'utérus ne représentent pondéralement que 2 à 3 p. Ioo de la matière sèche du blanc, celle-ci peut être utiliséc comme matériel de base pour exprimer l'évolution des teneurs en électrolytes de l'albumen après sa sortic du magnum. Nous pouvons d'autre part exprimer les concentrations par rapport à l'eau présente dans le blanc à chaque instant. Le rapprochement de ces deux modes d'expression permet d'avoir une idéc assez précise des mouvements d'eau et d'ions qui se produisent dans l'isthme et l'utérus.

Dans la suite du texte nous désignons par le symbole $\mathrm{X} m$ suivi des lettres (M), (I) ou (U) la teneur en l'élément $X$ de l'albumen en fin de magnum, d'isthme ou d'utérus (ceuf pondu) exprimées en méq $/ \mathrm{kg}$ de matière sèche. La notation $\mathrm{X}_{c}$ suivie des mêmes lettres est réservée aux concentrations exprimées en méq/1 d'eau (1).

La concentration théorique de chaque élément dans la solution ajoutéc à l'albumen in utero peut ainsi être calculée par la relation

dans laquelle :

$$
\mathrm{X} s . u .=-\frac{\mathrm{X} m}{(\mathrm{~L})-\mathrm{X} m} \frac{-\mathrm{I})}{\mathrm{E}_{2}-\mathrm{E}_{\mathbf{1}}}
$$

$\mathrm{X} s . u$. =: teneur théorique de la solution utérine (en méq/1).

$\mathrm{X} m(\mathrm{I})$ et $\mathrm{X} m(\mathrm{U})=$ teneurs (en méq/kg m. s.) de l'albumen de l'ouf retiré en fin d'isthme et de l'ouf pondu.

$\mathrm{E}_{\mathrm{g}}$ et $\mathrm{E}_{\mathrm{I}}=$ teneurs en cau (en $\mathrm{kg} / \mathrm{kg} \mathrm{m}$. s.) de l'albumcn des mêmes oufs.

Notons en fin quc dans l'cxpression des résultats relatifs aux effets de l'acidose mcitabolique les symboles $\Delta \mathrm{X} m$ et $\Delta \mathrm{X} e$ représentent, pour l'élément $\mathrm{X}$, les différences "aciclose "— "témoin " à un même niveau de l'oviducte.

\section{RÉSULTATS}

\section{Tenour en eall de l'albumen}

Nous l'avons exprimée en $\mathrm{g}$ eau/g $\mathrm{m}$. s. du blanc et avons reporté les valeurs correspondantes sur la figure I. Afin d'éliminer les variations possibles entre animaux,

(1) Ces deux modes d'expression sont liés par : avec

$$
\mathrm{X} n=\mathrm{X} c \times \mathrm{E}
$$

et

$\mathrm{X} m$ en még/kg m. s., Xe en méq/l d'eau

$$
\mathrm{E}=1 \mathrm{~d} \text { 'eau/kg m. s. pour chaque échantillon }
$$


nous avons également calculé les teneurs en eau des œufs " magnum " et "isthme " en p. Ioo de celle enregistrée dans l'œuf pondu par la même poule.

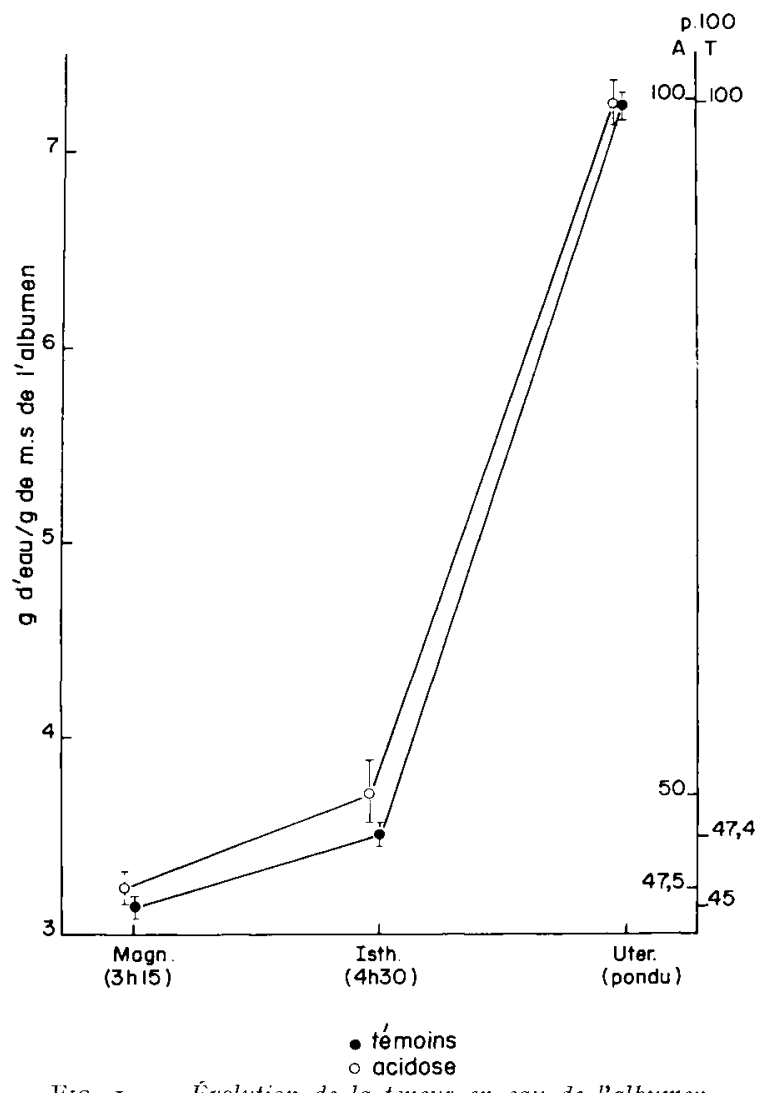

Fig. I. - Evolution de la teneur en eau de l'albumen au cours de la formation de l'cuf (1)

(') Sur chacune des figures, nous portons pour chaque stade ćtudié la valeur $\vec{x} \pm \frac{\sigma}{1 n}$

\section{- Animaux témoins.}

Entre la sortie du magnum et celle de l'isthme, l'albumen s'enrichit très légèrement en eau $(0,49 \mathrm{~g} / \mathrm{g} \mathrm{m}$. s., $p<0,0 \mathrm{I})$. Cette hydratation n'apparaît cependant plus significative lorsque nous comparons les teneurs en eau exprimées en p. Ioo de la valeur finale : 45 , $\mathrm{I} \pm 0,8$ contre $47,4 \pm \mathrm{I}, \mathrm{o}$ p. Ioo. Fin revanche, le passage de l'ouf dans l'utérus double la quantité d'eau présente dans l'albumen : 7,23 $\pm 0,08$ contre $3,5 \mathrm{I} \pm 0,06 \mathrm{~g}$ eaulg m. s. $(p<0,00 \mathrm{I})$.

- Action de l'acidose.

A aucun moment de la formation de l'œuf (magnum, isthme, ouf pondu), l'acidose n'agit sur la teneur en eau de l'albumen rapportée à la matière sèche (fig. I). L'écart le plus grand $(0,22 \mathrm{~g}$ eau/g m. s.), qui se situe à la fin de l'isthme, n'apparaît pas significatif. 


\section{Transfert des électrolytes à l'albumen}

L'évolution des valeurs $\mathrm{X}_{m}$ (méq/ $\mathrm{kg} \mathrm{m}$. s.) est représentée sur la figure 2 et celle des $\mathrm{X}_{e}$ (méq/1 eau) sur la figure 3 . Nous avons de plus rapporté sur la figure 4 les parts respectives du magnum, de l'isthme et de l'utérus dans l'apport des 4 éléments étudiés ; les concentrations théoriques calculées pour la solution utérine sont données par la figure 5 .

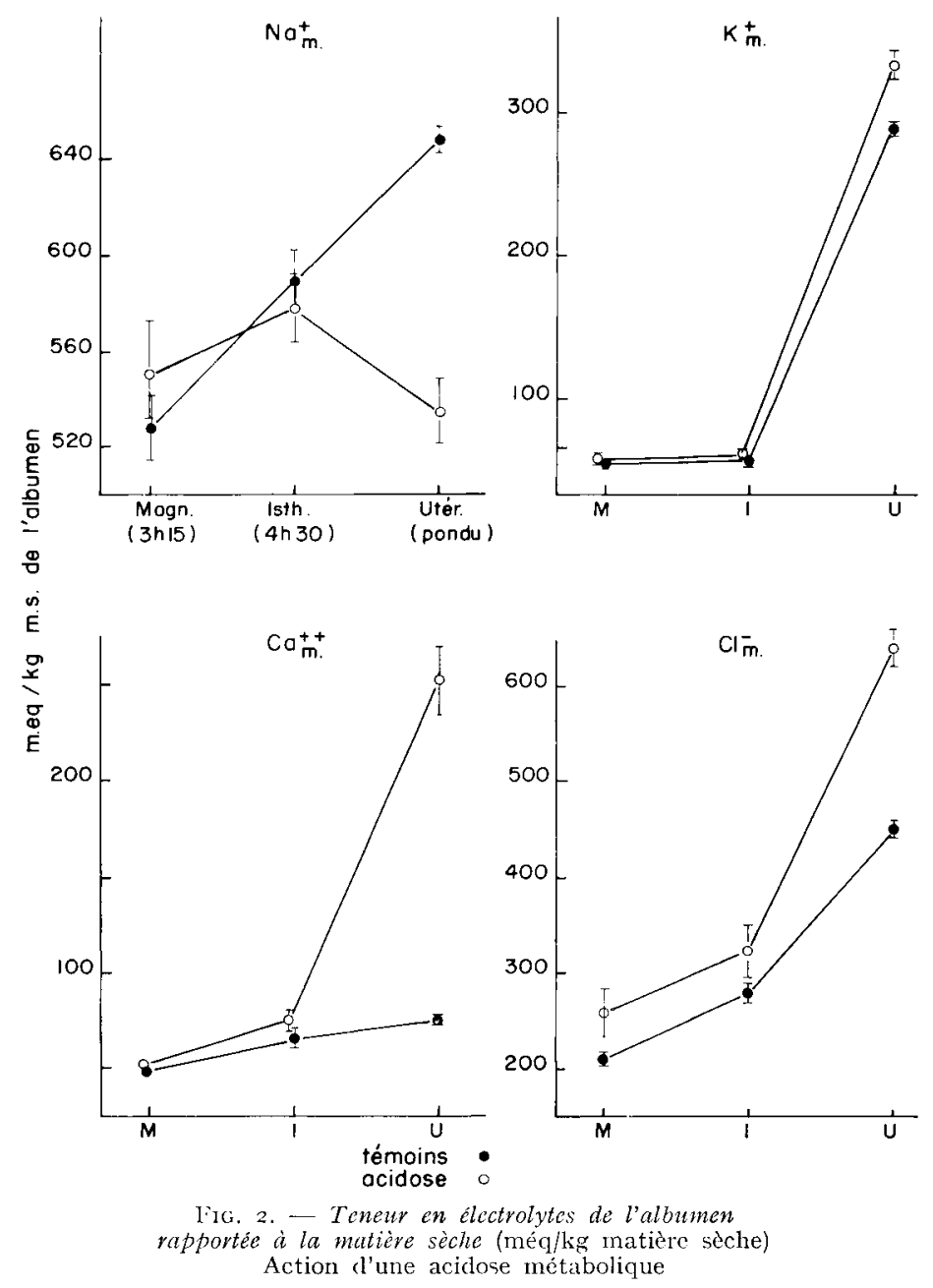

a) Sodium.

- Transfert normal (témoins). - Les trois valeurs successives de $\mathrm{Na}_{n}$ (fig. 2) et celles de la figure 4 montrent que la plus grande part du sodium total ( $8 \mathrm{I}, 6 \mathrm{p}$. Ioo) est apportée dans le magnum. Une fraction beaucoup plus faible (9,3 p. Ioo) mais significative $(p<0,00 r)$ est ensuite fournie par l'isthme ; la part de l'utérus enfin est équivalente à celle de 1 'isthme (9, I p. IOO, $p<0,00 \mathrm{r})$. 
Les teneurs en sodium rapportées à l'eau présente (fig. 3) sont identiques en fin de magnum et d'isthme ( $167,0 \pm \mathrm{I}, 0$ et $\mathrm{I} 67,7 \pm \mathrm{I}, \mathrm{I}$ méq/ 1 eau respectivement). Le léger apport de sodium par l'isthme constaté ci-dessus s'accompagne donc d'une hydratation correspondante. A 1'opposé 1'apport d'eau considérable effectué dans l'utérus entraîne une chute de $\mathrm{Na}_{e}$ de 75, I méq/1 eau $(p<0$,oor $)$.

Le calcul donne pour la solution ajoutée à l'albumen dans l'utérus (fig. 5) une teneur théorique en sodium de $\mathrm{I} 8,8 \mathrm{méq} / 1$, évidemment très inférieure à celle du blanc à sa sortie de l'isthme.

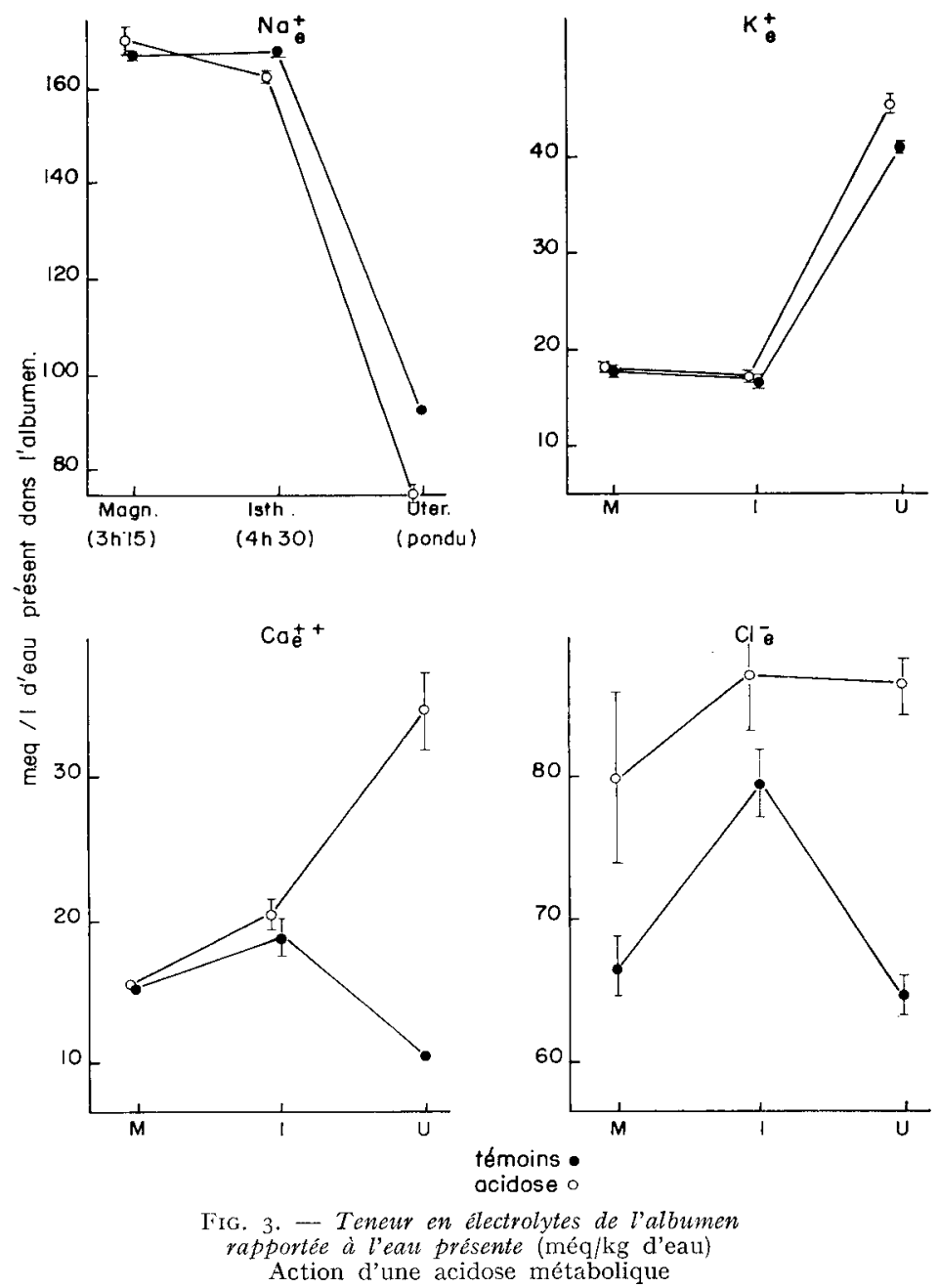

- Effet de l'acidose. - Les valeurs respectives de $\mathrm{Na}_{m}(\mathrm{M})$ et $\mathrm{Na}_{m}$ (I) observées dans les œufs témoins et dans ceux provenant des poules en acidose ne sont pas significativement différentes (fig. 2). C'est donc au niveau de 1'utérus que l'acidose induite par 1'ingestion de $\mathrm{NH}_{4} \mathrm{Cl}$ réduit la quantité totale de sodium présente dans 
l'albumen puisque la différence $\Delta \mathrm{Na}_{m}(\mathrm{U})=-\mathrm{II} 3,8 \mathrm{méq} / \mathrm{kg} \mathrm{m}$. s. est hautement significative $(p<0,00 \mathrm{I})$.

Il semble même qu'en cas d'acidose l'utérus soit le siège d'une " réabsorption " de sodium à partir de 1'albumen (fig. 4 et 5 ) mais la différence $\mathrm{Na}_{m}(\mathrm{I})-\mathrm{Na}_{m}(\mathrm{U})$ observée dans ces conditions (44,I méq $/ \mathrm{kg} \mathrm{m}$. s.) ne permet pas de conclure statistiquement. Disons simplement que l'acidose abolit l'apport de $\mathrm{Na}$ enregistré dans l'utérus des animaux témoins.

La valeur de $\mathrm{Na}_{e}$ n'est pas affectée par l'acidose au niveau du magnum (fig. 3) ; à l'opposé celle-ci entraîne en fin d'isthme une légère réduction de concentration $\left(\Delta \mathrm{Na}_{e}(\mathrm{I})=-5,03\right.$ méq $/ 1$ eau, $\left.p<0,05\right)$ résultant à la fois d'un apport un peu moindre de sodium et d'une hydratation légèrement plus élevée bien que ces deux variations, prises individuellement, ne soient pas apparues significatives (voir cidessus).

Le phénomène est beaucoup plus marqué dans l'utérus et se traduit dans l'albumen des cufs pondus, par $\Delta \mathrm{Na}_{e}(\mathrm{U})=-\mathrm{I} 7,9 \mathrm{méq} / 1$ eau $(p<0,00 \mathrm{I})$. La différence $\mathrm{Na}_{e}(\mathrm{I})-\mathrm{Na}_{e}(\mathrm{U})=88$ méq $/ 1$ eau $(p<$ o,oor $)$, supérieure à celle enregistrée chez les témoins (75, voir plus haut), montre également que la réduction in utero de la teneur en sodium? du blanc est accentuée par l'acidose métabolique.

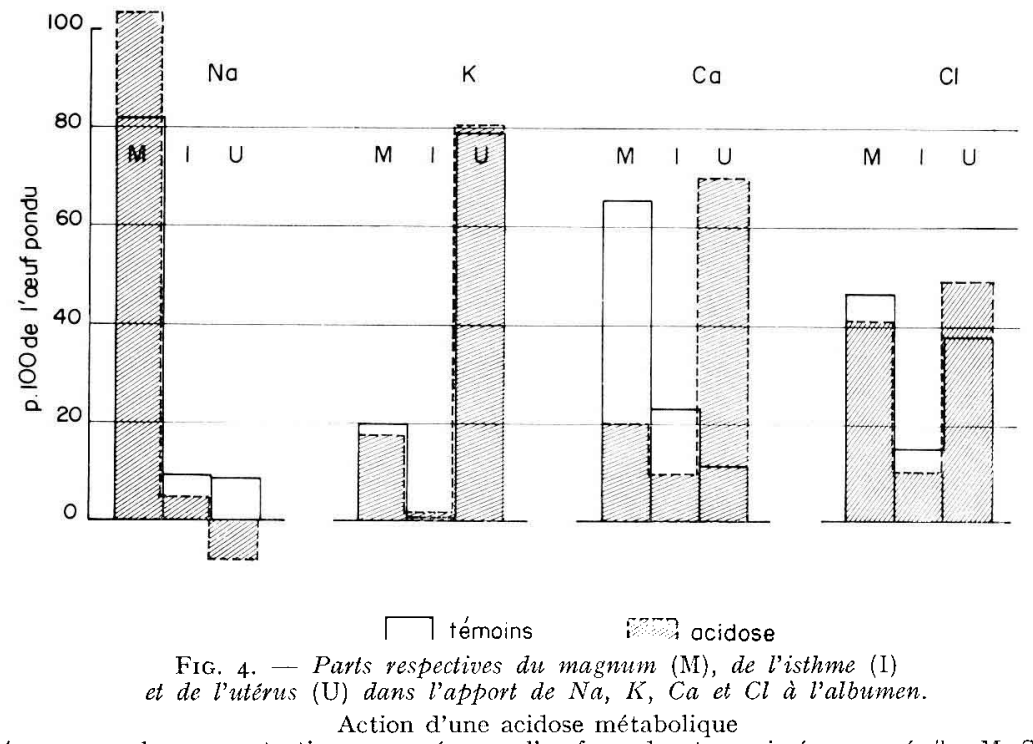

(en p. too des concentrations mesurées sur l'œuf pondu et exprimćes en méq/sı M. S.)

\section{b) Potassium.}

- Animaux témoins. - Les données des figures 2 et 4 montrent que l'apport de potassium est faible dans le magnum (I9,6 p. Ioo) et nul dans l'isthme. C'est 1'utérus qui fournit la plus grande part du potassium du blanc comme le montre la différence $\mathrm{K}_{m}(\mathrm{U})-\mathrm{K}_{m}(\mathrm{I})=230,2 \mathrm{méq} / \mathrm{kg} \mathrm{m} . \mathrm{s} .(p<0,00 \mathrm{I})$.

Les valeurs de $\mathrm{K}_{e}$ restent également inchangées entre la fin du magnum et celle de 1'isthme (fig. 3). Après le passage de l'œuf dans 1'utérus et malgré la très impor- 
tante hydratation qui s'y produit, $\mathrm{K}_{e}$ augmente de plus de Ioo p. Ioo $\left\langle\mathrm{K}_{e}(\mathrm{U})-\mathrm{K}_{e}(\mathrm{I})\right.$ $=24,4 \mathrm{méq} / 1$ eau $(p<0,00 \mathrm{I}))$. La concentration théorique de la solution apportée in utero est donc très supérieure à celle de l'albumen en fin d'isthme $(7 \mathrm{I}, 6$ contre I6,7 méq/1), fait qui sera discuté ultérieurement.

- Action de l'acidose. - Les écarts $\Delta \mathrm{K}_{m}(\mathrm{M})$ et $\Delta \mathrm{K}_{m}$ (I) non différents de zéro, montrent que la quantité totale de potassium apportée à l'albumen dans le magnum et l'isthme n'est pas modifiée par l'acidose (fig. 2). A l'opposé nous observons sous l'effet du traitement un accroissement de la teneur finale du blanc en $\mathrm{K}: \Delta \mathrm{K}_{m}(\mathrm{U})$ $=44,3 \mathrm{méq} / \mathrm{kg} \mathrm{m}$. $\mathrm{s} .(p<0$, oor $)$. C'est donc uniquement au niveau de l'utérus que l'apport de potassium est affecté par les variations de l'équilibre acido-basique de l'animal.

Nous aboutissons à la même conclusion en considérant les valeurs successives de $\mathrm{K}_{e}$ qui ne varient, sous l'effet de l'acidose, que dans 1'œuf pondu $\left(\Delta \mathrm{K}_{e}(\mathrm{U})=4,7 \mathrm{méq} / 1\right.$ eau $p<0,00 I$ ) (fig. 3). Le transfert utérin de potassium vers l'albumen, déjà très important dans les conditions normales, semble donc être encore légèrement accru par ce traitement.

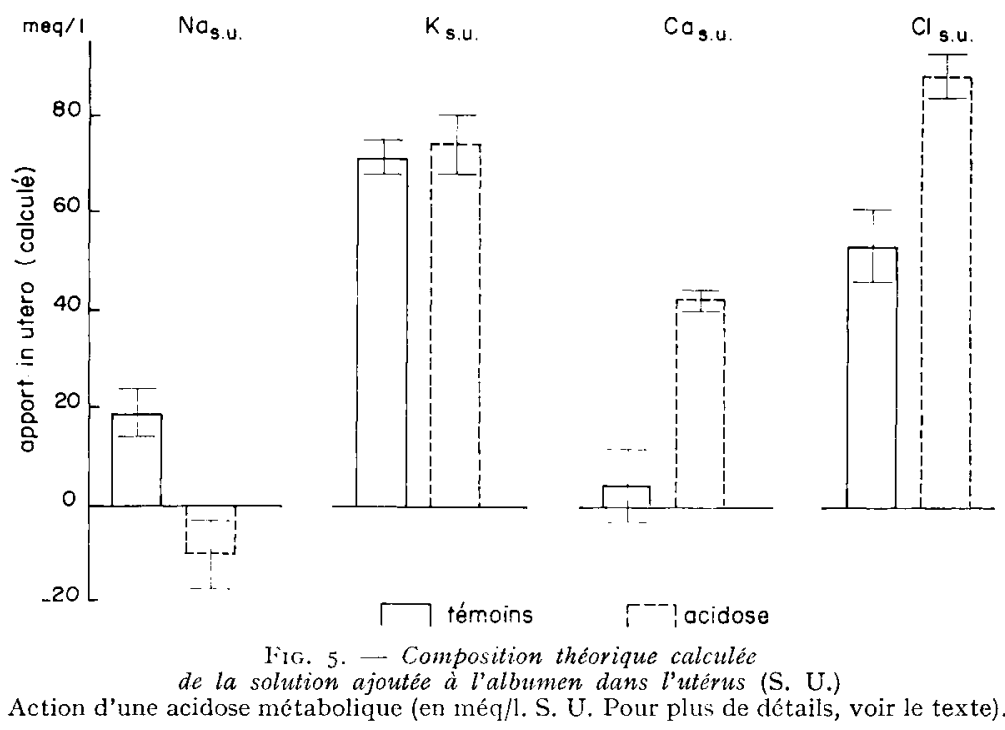

c) Calcium.

- Dans les conditions d'équilibre acido-basique normales (témoins) l'apport de calcium à l'albumen est à la fois le fait du magnum, de l'isthme et, peut-être, de l'utérus (fig. 2 et 4 ). Le magnum est le plus important avec 65,6 p. Ioo du Ca total; l'apport de l'isthme (23, I p. roo) n'est pas négligeable et se traduit par la différence $\mathrm{Ca}_{m}(\mathrm{I})-\mathrm{Ca}_{m}(\mathrm{M})=\mathrm{I} 7,3 \mathrm{méq} / \mathrm{kg} \mathrm{m} . \mathrm{s} .(p<0, \mathrm{OOI})$. L'utérus enfin contribue probablement à un faible enrichissement du blanc en Ca qui n'apparaît cependant pas significatif ici.

Du fait du transfert effectif de calcium dans l'isthme, $\mathrm{Ca}_{e}$ augmente durant le passage de 1'œuf à ce niveau $\left(\mathrm{Ca}_{e}(\mathrm{I})-\mathrm{Ca}_{e}(\mathrm{M})=3,3\right.$ méq $/ 1$ eau, $p<0$,oor); en 
revanche, le très faible apport utérin ne suffit pas à maintenir constante la teneur en Ca du blanc qui diminue de 8,3 méq/1 d'eau pendant le passage de l'œuf dans l'utérus $(p<0$,oor) (fig. 3).

- Action de l'acidose. - Le calcium est sans conteste l'élément dont le transfert est le plus affecté par l'état d'acidose des pondeuses. Au niveau du magnum il n'apparaît cependant pas encore de différences dues au traitement que l'on considère la quantité totale de $\mathrm{Ca}$ présente $\left(\mathrm{Ca}_{m}\right.$, fig. 2) ou sa concentration par rapport à l'eau de l'albumen ( $\mathrm{Ca}_{e}$, fig. 3$)$. Il en est de même à la fin de l'isthme où les écarts $\Delta \mathrm{Ca}$ ne sont pas significatifs alors que nous enregistrons dans l'albumen des œufs pondus " sous acidose " une teneur en calcium plus de trois fois supérieure à celle des œufs témoins.

Cette modification très importante du transfert utérin de Ca peut être chiffrée par les deux valeurs $\Delta \mathrm{Ca}_{m}(\mathrm{U})=\mathrm{I} 75,5 \mathrm{méq} / \mathrm{kg} \mathrm{m}$. s. et $\Delta \mathrm{Ca}_{e}=24,0 \mathrm{méq} / 1$ eau ( $p<0$,OoI dans les deux cas). Il en résulte que, lors d'une acidose métabolique, l'utérus devient le lieu principal d'enrichissement de l'albumen en calcium $(69,9$ p. roo contre II,2 p. Ioo chez les témoins) bien que la quantité initiale apportée dans le magnum ne soit pas modifiée (fig. 4). Dans ces mêmes conditions, $\mathrm{Ca}_{e}$ ne diminue plus dans l'utérus mais subit au contraire une forte augmentation (fig. 3).

Ce phénomène se retrouve en considérant la concentration théorique de l'apport utérin qui passe de 4,4 (chez les témoins) à 42,5 méq $/ 1$ de calcium chez les animaux recevant $\mathrm{NH}_{4} \mathrm{Cl}$ (fig. 5).

d) Chlore.

Comme le sodium et le calcium, le chlore est apporté à l'albumen de l'œuf dans le magnum, l'isthme et 1'utérus (fig. 2 et 4). Bien qu'assez faible, la contribution de l'isthme n'est pas négligeable ( $15,2 \mathrm{p}$. Ioo du $\mathrm{Cl}$ total); elle est cependant inférieure à celle de $1^{\prime}$ utérus ( $38 \mathrm{p}$. roo) qui se rapproche de celle du magnum (46,7 p. Ioo) et se traduit par la différence : $\mathrm{C}_{m}(\mathrm{U})-\mathrm{C}_{m}(\mathrm{I})=\mathrm{I} 72 \mathrm{méq} / \mathrm{kg} \mathrm{m} . \mathrm{s} .(p<0,00 \mathrm{I})$. Ainsi l'enrichissement de l'albumen en chlore s'effectue suivant un processus intermédiaire entre celui du sodium (où le magnum joue le plus grand rôle) et celui du potassium (dans lequel l'utérus est prépondérant).

La teneur en chlore de l'albumen rapportée à l'eau présente $\left(\mathrm{Cl}_{e}\right)$ s'accroit dans l'isthme $(+\mathrm{I} 2,9 p<0, \mathrm{OI})$ ptiis diminue dans l'utérus $(-\mathrm{I} 4,8 p<0, \mathrm{OOI})$ (fig. 3 ).

- Action de l'acidose. - Nous enregistrons en fin de magnum dans les œufs provenant des animaux en acidose, une légère augmentation de la teneur en chlore de l'albumen: $\Delta \mathrm{Cl}_{m}(\mathrm{M})=5 \mathrm{O}(p=0$,or $)$ et $\Delta \mathrm{Cl}_{e}(\mathrm{MI})=\mathrm{I}_{3,2}(p<0,05$ (fig. 2). Le chlore est donc le seul élément étudié pour lequel le rôle du magnum est modifié par l'ingestion de $\mathrm{NH}_{4} \mathrm{Cl}$.

Ėn fin d'isthme, un léger avantage subsiste au profit des œufs " acidose " mais les écarts ne sont pas significatifs. La plus grande part de l'accroissement de teneur en chlore observé dans les œufs pondus et attribuable à 1'acidose, est encore le fait de 1'utérus $\left(\Delta \mathrm{Cl}_{m}(\mathrm{U})=\mathrm{I} 79,7 p<0,00 \mathrm{I}\right)$. Il s'ensuit que $\mathrm{Cl}_{e}$ ne diminue plus à ce niveau mais se maintient à la valeur observée en fin d'isthme (fig. 3). Notons enfin que, dans ces mêmes conditions, l'utérus devient légèrement prépondérant dans l'apport de chlore à l'albumen $(48,6 \mathrm{p}$. Ioo du $\mathrm{Cl}$ total contre $4 \mathrm{r}, 3 \mathrm{p}$. Ioo pour le magnum) (fig. 4). 


\section{DISCUSSION}

L.es résultats rapportés ici sont de deux ordres et concernent d'une part l'étude du transfert de $\mathrm{Na}, \mathrm{K}$, Ca et $\mathrm{Cl}$ à l'albumen de l'œuf chez des animaux normaux et d'autre part les modifications de ces mêmes transferts lorsque les pondeuses reçoivent un régime supplémenté avec $3 \mathrm{p}$. Ioo de $\mathrm{NH}_{4} \mathrm{Cl}$ qui entraîne l'apparition d'une acidose métabolique sévère (SAUveur, Ig69). Disons d'abord quelques mots des résultats obtenus chez les animaux témoins.

Nous retrouvons en premier lieu que, lors du passage de l'œuf dans l'utérus, la quantité d'eau présente dans l'albumen est multipliée par deux. Ce processus, fondamental pour la constitution finale de l'œuf et le développement de l'embryon, a été maintes fois décrit depuis les premiers travaux de PEARL et CuRTIS (I9I2) sans avoir reçu d'explication théorique satisfaisante; nous y reviendrons très brièvement en fin de discussion.

Draper (I966 $a$ et $b$ ) montre que la teneur de l'albumen en sodium et calcium (exprimée en méq/l eau) diminue progressivement depuis le magnum jusque dans l'œuf pondu tandis que sa teneur en potassium augmente dans l'utérus. Nos résultats, bien que d'amplitude souvent différente, sont dans l'ensemble en excellent accord avec ceux de cet auteur; nous trouvons cependant que, lors du passage de 1'œuf dans l'isthme, la teneur de l'albumen en calcium continue de croître, observation en accord avec la forte concentration en calcium trouvée dans la paroi de 1'isthme par TAYLOR et HERTELENDY (I960) et MisRA et KEMENY (I964). Nous montrons également, à la suite de DRAPER, que les quantités totales de $\mathrm{Na}$ et $\mathrm{Ca}\left(\mathrm{Na}_{m}\right.$ et $\left.\mathrm{Ca}_{m}\right)$ augmentent en permanence depuis le magnum jusqu'à l'utérus mais nous ne trouvons, quant à nous, aucun apport de potassium au niveau de l'isthme.

L,es mesures effectuées ici ne sont évidemment que des "photographies " du contenu minéral du blanc à des instants précis et ne permettent pas de savoir si certains ions sont ou non réabsorbés dans l'utérus. La possibilité de tels transferts a été évoquée par HoOver et SMiTh (I958) et DrAPLR (I966 a) et reste valable à la suite de cette étude; nous y reviendrons dans quelques instants.

Remarquons également que les concentrations théoriques calculées pour la solution ajoutée à l'albumen dans l'utérus ne préjugent en rien de la composition du fluicle utérin lui-même. Ces deux séries de données apparaissent d'ailleurs fort différentes si l'on se réfère aux résultats de BEAdis, ConRAD et ScoT'T (I938) et EI. JAck et LAKE (I967) rapportés au tableau $I$.

TABIEAU I

Composition du fluide utérin au moment du "plumping" (en méq/l)

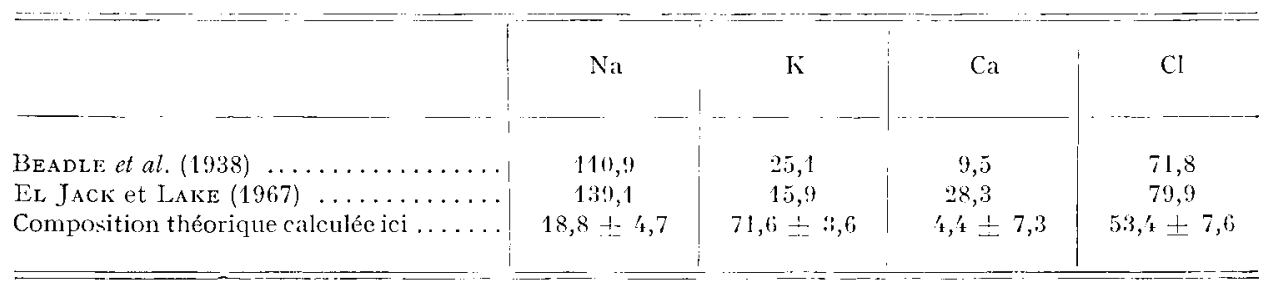


Il est même remarquable, surtout en ce qui concerne les ions $\mathrm{Na}^{+}$et $\mathrm{K}^{+}$, que la concentration théorique calculée soit si éloignée de celle du fluide utérin. Ainsi ce dernier apparaît très riche en sodium alors que l'albumen ne s'enrichit que très peu en cet élément dans l'utérus tandis que la faible teneur en potassium du fluide utérin doit aller de pair avec un transfert énorme de même élément vers l'albumen. Il semble donc impossible d'expliquer les mouvements de ces deux ions par de simples phénomènes de diffusion passive, conclusion qui confirme celle de SMrTH et al. (I954) et HOOVER et SMITH (I958).

Notons enfin que nous pouvons compléter les résultats de DrAPER en y ajoutant ceux obtenus ici sur le transfert du chlore qui semble se faire à peu près également dans le magnum et 1'utérus avec une contribution moindre de l'isthme.

Enn résumé nous pouvons dire qu'au moment où l'œuf pénètre dans l'utérus, une grande part du contenu inorganique de l'albumen est déjà déposée sans oublier cependant que $80 \mathrm{p}$. Ioo du potassium et $3^{8} \mathrm{p}$. Ioo du chlore seront transférés dans cet organe; il convient par ailleurs d'y ajouter l'apport des ions $\mathrm{HCO}_{3}-$ (BEAdic, ConRAD et ScotT, I938) et de 70 p. roo de l'iode (Oshima et al., I963).

\section{Action d'une acidose métabolique sur le transfert des minéraux}

L'adjonction au régime des pondeuses de chlorure d'ammonium au taux de 3 p. Ioo modifie grandement la teneur en éléments inorganiques de l'albumen de l'œuf pondu, à savoir: sodium - I9 p. Ioo, potassium + II p. Ioo, calcium +225 p. Ioo, chlore +34 p. Ioo, valeurs proches de celles rapportées en introduction.

Le résultat le plus intéressant réside à notre avis dans le fait que les différentes parties de l'oviducte sont très inégalement touchées par l'état d'acidose de la pondeuse. C'est ainsi que l'accroissement de teneur du blanc en $\mathrm{K}$ et Ca est uniquement dû aux transferts effectués dans l'utérus. Ce fait peut paraître logique dans le cas du potassium dont $80 \mathrm{p}$. Ioo sont normalement apportés à ce niveau; il n'en est pas de même pour le calcium dont $89 \mathrm{p}$. Ioo sont, en l'absence de traitement, fournis par le magnum et 1 'isthme. I1 est de plus remarquable que 1'acidose multiplie par Io la teneur théorique en $\mathrm{Ca}$ de la solution ajoutée à l'albumen dans 1'utérus alors que la quantité apportée par le magnum reste, dans les mêmes conditions, rigoureusement constante.

Comme nous ignorons actuellement si l'acidose entraîne ou non un accroissement de la teneur en calcium du fluide utérin réel, deux hypothèses peuvent être envisagées pour expliquer ce transfert exceptionnel de Ca in utero: soit la teneur du fluide utérin est inchangée et le Ca de l'albumen était en fait destiné à la formation de la coquille; on sait en effet que des acidoses métaboliques réduisent le dépôt de $\mathrm{CaCO}_{3}$ sur la coquille mais que la fraction carbonate semble en être le principal responsable (MoNGIN, Ig68) ; soit, cleuxième hypothèse, l'utérus, en présence d'une acidose générale, met en jeu des mécanismes identiques à ceux du néphron : MoNGin (I970) démontre en effet qu'une perfusion de $\mathrm{HCl}$ chez la poule provoque un accroissement d'excrétion rénale du calcium de $5_{5} \mathrm{p}$. Ioo.

Les travaux d'ABELs (I936) et de SMith et al. (I954) tendent par ailleurs à démontrer que le calcium pourrait être lié aux protéines du blanc; nos résultats ne permettent pas de soutenir rigoureusement cette hypothèse mais il est en effet pos- 
sible qu'une telle liaison existe au niveau du magnum puisque la quantité de calcium fournie par cet organe reste constante malgré le traitement appliqué. A 1'opposé i1 est probable que le calcium ajouté à l'albumen in utero l'est sous forme ionisée.

En ce qui concerne les apports de sodium et de chlore, l'utérus est encore la partie de l'oviducte la plus affectée par l'ingestion dê chlorure d'ammonium ; nous avons vu cependant que l'acidose exerce également une très légère action sur la teneur en $\mathrm{Na}$ du blanc (en méq/1 d'eau) au niveau de l'isthme. Le chlore enfin paraît être le seul élément dont l'apport au niveau du magnum soit modifié par le traitement, résultat qui peut s'expliquer simplement par une augmentation de la chlorémie. Nous avons en effet montré (SAUVEUR, Ig6g) qu'après ingestion continue de 3 p. IOo de $\mathrm{NH}_{4} \mathrm{Cl}$, la chlorémie s'accroît de Io p. IoO environ alors que la calcémie et la kaliémie restent constantes; dans les mêmes conditions la natrémie chute de 4 p. Ioo environ ce qui est peut-être en rapport avec les phénomènes observés au niveau de l'isthme.

Notons que, sous l'effet de l'acidose, la quantité de sodium apportée par l'utérus diminue en même temps qu'augmente celle de potassium ; ceci pourrait être un argument en faveur d'un échange $\mathrm{Na}-\mathrm{K}$ dans l'utérus lié à une réabsorption de sodium déjà évoquée (DRAPER, Ig66 a). (I)

Rappelons cependant qu'à aucun moment de la formation de l'œuf nous n'avons trouvé, sous l'action du chlorure d'ammonium, de variations de la teneur en eau du blanc rapportée à la matière sèche ; ce fait confirme et complète des résultats antérieurs (HUNT et AITKEN, I962; SAUVEUR, I970) mais implique que les teneurs en eau et en sodium de l'albumen ne sont pas rigoureusement liées : le mécanisme responsable de l'apport massif d'eau in utero réclame donc encore de nombreux éclaircissements.

\section{RÉSUMÉ}

Nous avons étudié les contributions respectives du magnum, de l'isthme et de l'utérus dans l'enrichissement en eau et en électrolytes $(\mathrm{Na}, \mathrm{K}$, Ca et $\mathrm{Cl}$ ) de l'albumen de l'ouf chez des animaux témoins et chez d'autres recevant 3 p. Ioo de $\mathrm{NH}_{4} \mathrm{Cl}$ dans leur aliment. A chaque niveau de l'oviducte, les teneurs en chaque élément ont été calculées par rapport à la matière sèche $\left(C_{m}\right.$, fig. 2) et à l'eau de l'albumen ( $\mathrm{C}_{e}$, fig. 3). Nous en avons déduit la part respective de chaque segment (fig. 4) et la composition théorique de la solution apportée dans l'utérus (fig. 5).

Il apparaît que les trois parties de l'oviducte apportent des électrolytes à l'albumen mais que l'isthme joue un rôle secondaire. Chez les animaux témoins, le magnum est prépondérant pour l'apport de $\mathrm{Na}$ et $\mathrm{Ca}$, l'utérus pour celui du potassium. Ces résultats confirment dans l'ensemble ceux déjà connus.

L'acidose due à l'ingestion de $\mathrm{NH}_{4} \mathrm{Cl}$ altère grandement la composition de l'œuf pondu ; pour $\mathrm{Na}, \mathrm{K}$, Ca l'utérus est le seul responsable des modifications enregistrées ; pour le chlore le transfert effectué dans le magnum est légèrement accru mais la modification essentielle se situe également dans l'utérus. Sous l'effet de l'acidose $\mathrm{Cl}_{e}$ et $\mathrm{Ca}_{e}$ augmentent pendant la traversée de l'utérus qui devient dans ces conditions l'organe prépondérant.

Le fait que la contribution du magnum ne soit pas (ou très peu pour $\mathrm{Cl}$ ) affectée par l'acidose fait penser qu'il doit exister deux modes de transport différents des minéraux dont le second (dans l'utérus) serait le seul sensible aux modifications de l'équilibre acido-basique général.

(I) Nous avons, pendant la publication de ce texte, démontré effectivement que l'apport de $\mathrm{K}^{+}$ in utero est accompagné d'une réabsorption de $\mathrm{Na}^{+}$et que les deux flux sont égaux en valeur absolue (voir Mongin et SAUveUr, I970).

Annales de Biologie animale. -- I97o. 


\section{SUMMARY}

\section{ACTION OF A METABOLIC ACIDOSIS ON IONS TRANSFERS IN HEN'S OVIDUCT}

We have studied the respective contributions of magnum, isthmus and uterus in water and electrolytes ( $\mathrm{Na}, \mathrm{K}, \mathrm{Ca}$ and $\mathrm{Cl}$ ) enrichment in egg albumen of control animals and animals fed 3 per cent $\mathrm{NH}_{4} \mathrm{Cl}$. In oviduct each element level has been calcutated plotted to dry matter $\left(C_{m}\right.$, fig. 2) and to albumen water $\left(C_{e}\right.$, fig. 3). We have deduced the respective parts of each segment (fig. 4) and the theoretical composition of the solution provided to uterus (fig. 5).

It appears that the three parts of oviduct provide electrolytes to albumen, but that isthmus plays but a minor role. In control animals, magnum plays the leading part in $\mathrm{Na}$ and $\mathrm{Ca}$ supply, and uterus in potassium supply. On a whole, these results confirm results already known.

Acidosis due to $\mathrm{NH}_{4} \mathrm{Cl}$ ingestion greatly affects laid egg composition; uterus is the only organ involved in $\mathrm{Na}, \mathrm{K}$ and $\mathrm{Ca}$ registered modifications; chloride transfer to magnum is slightly increased, but the main modification also occurs in uterus. Under the effect of acidosis, $\mathrm{Cl}_{w}$ and $\mathrm{Ca}_{w}$ increase during passage in uterus ; in these conditions, uterus becomes the leading organ.

Magnum contribution being not effected by acidosis (except a small impact on Cl), we may think that two different forms of minerals transport exist; the second form (in uterus) would be the only one affected by modifications in general acid-base balance.

\section{RÉFÉRENCES BIBLIOGRAPHIQUES}

Abels J.-C., 1936. J. Amer. Chem. Soc., 58, 2609-26ro. Cited by Romanoff A. L., Romanoff A. I. 1949. The Avian Egg, p. 355, John Wiley and Sons Inc., New York.

Beadle B. W., Conrad R. M., Scott H. M., I938. Composition of the uterine secretion of the domestic fowl. Poultry Sci., 17, 498-504.

DRAPER M. H., I 966 a. The accumulation of water and electrolytes in the egg of the hen. In HoRTON-SMITH C., Amoroso B. C., Physiology of the domestic fow (B. E. M. B. Symposium no 1), 63-74, Oliver and Boyd, Edinburgh and London.

Draper M. H., ig66 b. Le transport des minéraux à l'albumen de l'ceuf de poule. Proc. 13th World's Poultry Congr., 338-34I.

EL JACK M. H., LAKE P. E., I967. The content of the principal inorganic ions and carbon dioxide in uterine fluids of the domestic hen. J. Reprod. Fert., 13, I27-I 32.

Hoover G. N., Sмith A. H., 1958. Secretion of fluid by the shell gland of the laying hen. Poultry Sci., $37,467-47 \mathrm{r}$.

Hunt J. R., I964. Electrolytes changes in albumen on feeding $\mathrm{NH}_{4} \mathrm{Cl}$. Poultry Sci., 43, I33I (Abstr.).

Hunt J. R., Aitken J. R., Ig62. The effect of ammonium and chloride ions in the diet of hens on egg shell quality. Poultry Sci., 41, 434-438.

MIsRA M. S., KemenY A., I964. Studies on the oviduct and serum of fowls. I. Oxygen uptake, alkaline phosphatase activity, concentrations of phosphorus, calcium and magnesium in adult Hungarian Yellow hens. Acta V'et. Hung., 14, 387-397.

Mongin P., I968. Role of acid-base balance in the physiology of egg shell formation. World's Poult. Sci. J., 24, 200-230.

Mongrn P., I970. Communication personnelle.

Mongin P., Sauveur B., r97o. Composition du fluide utérin et de l'albumen durant le séjour de l'ouf dans l'utérus chez la poule domestique. C. R. Acad. Sci., Sêr. D. 270, I

Oshima M., Suzuki M., Nozaki H., I963. On the albumen secretion in the laying hen II. The addition of mineral elements into the albumen during the passage of the egg in the oviduct, in special reference to I3I I. Summ. rep. Bull. Vation. Inst. Anim. Industr. 1, 18-19.

Pearl R., Curtis M. R., I9:2. Studies on the physiology of reproduction in the domestic fowl. V. Data regarding the physiology of the oviduct. J. Exp. Zool, 12, 99-I32.

Sauvetr B., rg69. Acidoses métaboliques expérimentales chez la Poule pondeuse. I. Action sur l'équilibre acido-basique du sang et l'excrétion rénale des électrolytes. Ann. Biol. anim. Bioch. Biophys. $9(3), 379-39 \mathrm{I}$.

Sauveur B., I970. Acidoses métaboliques expérimentales chez la Poule pondeuse. II. Action sur la composition minérale de l'albumen de l'cuf. Ann. Biol. anim. Bioch. Biophys. 10 (1), 81-Ioo.

Scots H. M., Hughes J. S., Warren D. C., I937. Augmentation of nitrogen to the egg white after formation of the shell membranes in the fowl. Poultry Sci., 16, 53-6r. 
Smith A. H., Wilson W. O., Brown J. G., r954. Composition of eggs from individual hens maintained under controlled environments. Poultry Sci., 33, 898-9o8.

TAyloR T. G., Hertelendy F., r960. Parallel distribution of calcium and citric adic in the oviduct of the hen. Nature, 187, 244-245. 